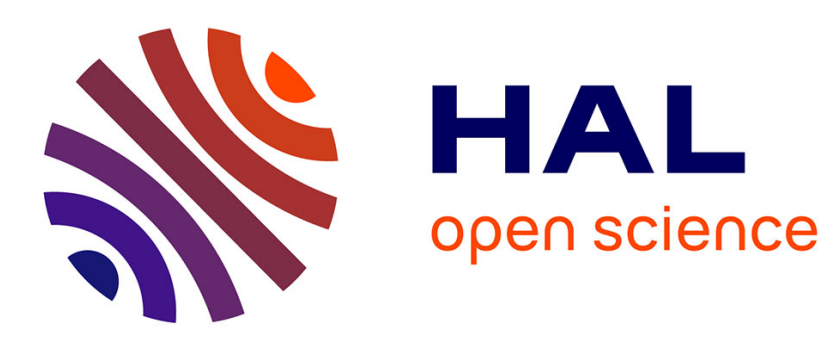

\title{
KnBest - A Balanced Request Allocation Method for Distributed Information Systems
}

\author{
Jorge-Arnulfo Quiane-Ruiz, Philippe Lamarre, Patrick Valduriez
}

\section{To cite this version:}

Jorge-Arnulfo Quiane-Ruiz, Philippe Lamarre, Patrick Valduriez. KnBest - A Balanced Request Allocation Method for Distributed Information Systems. Database Systems for Advanced Applications

(DASFAA), Apr 2008, Bangkok, Thailand. inria-00374835

\section{HAL Id: inria-00374835 \\ https://hal.inria.fr/inria-00374835}

Submitted on 9 Apr 2009

HAL is a multi-disciplinary open access archive for the deposit and dissemination of scientific research documents, whether they are published or not. The documents may come from teaching and research institutions in France or abroad, or from public or private research centers.
L'archive ouverte pluridisciplinaire $\mathbf{H A L}$, est destinée au dépôt et à la diffusion de documents scientifiques de niveau recherche, publiés ou non, émanant des établissements d'enseignement et de recherche français ou étrangers, des laboratoires publics ou privés. 


\title{
$\mathrm{K}_{n}$ Best - A Balanced Request Allocation Method for Distributed Information Systems ${ }^{\star}$
}

\author{
Jorge-Arnulfo Quiané-Ruiz ${ }^{\star \star}$, Philippe Lamarre, and Patrick Valduriez \\ INRIA and LINA \\ Université de Nantes \\ 2 rue de la houssinière, 44322 Nantes Cedex 3, France \\ \{Jorge.Quiane, Philippe.Lamarre\}@univ-nantes.fr, Patrick.Valduriez@inria.fr
}

\begin{abstract}
In large-scale distributed information systems, providers are typically autonomous, i.e. free to leave the system at will or to perform certain requests. In this context, request allocation is critical for the efficient system's operation. However, most methods used in distributed information systems aim at maximizing overall system performance (throughput and response times) by allocating requests to the most efficient providers, without considering providers' autonomy. In this paper, we propose a balanced request allocation method, $K_{n}$ Best, which considers providers' autonomy in addition to load balancing. Our method is general and simple, so that it can be easily incorporated in existing distributed information systems. We describe the implementation of $K_{n}$ Best in different scenarios. Finally, we give an experimental evaluation which shows that $K_{n}$ Best significantly outperforms traditional request allocation methods.
\end{abstract}

\section{Introduction}

We consider distributed information systems that are dynamic and provide access to a large number of information providers. Providers can be fairly autonomous, i.e. free to leave the system at will and to express their intentions for performing requests. Their intentions can stem from combining their preferences with other important factors such as their strategies.

In this context, allocating requests to providers must maximize overall system performance (throughput and response times). The traditional solution, used in mediator systems $[6,14,17]$, is to reduce the overloading of providers as much as possible so that load balancing is increased. However, preserving the providers' intentions when performing request allocation is equally important to keep providers happy and the system stable. With the traditional solution, providers can become unsatisfied when their intentions are not met and simply quit, resulting in an unstable system. Therefore, request allocation should

\footnotetext{
* Work partially funded by ARA "Massive Data" of the French ministry of research (projects MDP2P and Respire) and the European Strep Grid4All project.

** This author is supported by the Mexican National Council for Science and Technology (CONACyT).
} 
also take into account providers' intentions. This is a timely problem with the deployment of web services and service-oriented architectures.

Providers' intentions are dynamic and depend on the providers' context. For instance, some providers may desire to perform specific requests at some time, but not at another time. Furthermore, providers can be heterogeneous in terms of capacity and data. Heterogeneous capacity means that some providers are more powerful than others and can treat more requests per time unit. Data heterogeneity means that different providers provide different data and thus produce different results for the same request.

In this paper, we address the request allocation problem by considering providers' intentions in addition to load balancing. We propose a balanced request allocation method, called $K_{n}$ Best. It is inspired by the two random choices paradigm which has proven useful for dynamically assigning tasks to providers $[10,20,21]$. Our method is general and simple, so that it can be easily incorporated in existing distributed information systems. It generalizes traditional methods and can be adapted to the application by varying several parameters. We describe the implementation of $K_{n} B e s t$ in different scenarios. Finally, we give an experimental evaluation which compares $K_{n}$ Best to traditional request allocation methods. We show that, with autonomous information providers in the system, our method significantly outperforms these methods

The rest of this paper is organized as follows. In Section 2, we define the system model and introduce some notations. In Section 3, we present the $K_{n} B e s t$ method. In Section 4, we give the experimental evaluation of $K_{n}$ Best. In Section 5 , we discuss related works. Section 6 concludes.

\section{Model and Notations}

We consider a system consisting of a set of mediators, $M$, of a set of consumers, $C$, and of a set of providers, $P$. These sets are not necessarily disjoint, i.e. we can have $M \cap C \cap P \neq \emptyset$. Requests are formulated in a format abstracted as a triple $q=\langle c, d, n\rangle$ such that $q . c \in C$ is the identifier of the consumer that has issued the request, q.d is the description of the task to be done, and $q . n \in \mathbb{N}^{*}$ is the number of providers to which the consumer wishes to allocate its request. Consumers send their requests to a mediator $m \in M$ which allocates each incoming request $q$ to the $q . n$ providers in $P_{q}$, where $P_{q}$ denotes the set of providers that can treat $q$. We use $N_{q}$ for denoting $\left\|P_{q}\right\|$, or simply $N$ for the sake of simplicity when there is no ambiguity on $q$. In the case where $q \cdot n>N_{q}$, the consumer $q . c$ will get $N_{q}$ results instead of $q . n$. We only consider the arrival of feasible requests, that is, those requests for which $P_{q} \neq \varnothing$.

Request allocation of some feasible request $q$ among the providers which are able to deal with $q$ is defined as a vector All $\overrightarrow{o c}$ or $A l l \overrightarrow{o c}_{q}$ when there is an ambiguity on $q$ (see Definition 1 ). The set of providers such that $A l l \overrightarrow{o c}[p]=1$ is noted $\widehat{P_{q}}$.

Definition 1. Request Allocation

The allocation of request $q$ among the providers in $P_{q}$ is a vector Alloc of length 
$N$ such that: $\forall p \in P_{q}, A l l \overrightarrow{o c}[p]=\mid \begin{array}{ll}1 & \text { if provider } p \text { gets the request } \\ 0 & \text { otherwise }\end{array}$ with $\sum_{p \in P_{q}} \operatorname{All} \overrightarrow{o c}[p]=\min (q . n, N)$

Each provider $p \in P$ has a finite capacity, $\operatorname{cap}(p)>0$, for performing feasible requests. The capacity of a provider denotes the number of computational units that it can have. Similarly, each feasible request $q$ has a $\operatorname{cost} \operatorname{cost}_{p}(q)>0$, that represents the computational units that $q$ consumes at $p$. We define provider utilization as follows.

\section{Definition 2. Provider Utilization}

Let $Q_{p}$ denote the set of requests that have been allocated to $p$ but have not already been treated at time $t$ (i.e. the pending requests at $p$ ). The utilization of a given provider $p \in P$ at time $t, U_{t}(p)$, is defined as the computational units that $Q_{p}$ consumes at $p$

$$
U_{t}(p)=\frac{\sum_{q \in Q_{p}} \operatorname{cost}_{p}(q)}{\operatorname{cap}(p)}
$$

Providers are free to express their intentions for performing each feasible request $q$, denoted by the $P I_{p}(q)$ function whose values are between $-\infty$ and 1 , and where $p$ denotes a given provider. If the intention value is positive, the greater it is, the greater the desire for performing requests. If the intention is negative, the smaller it is, the greater the refusal for performing requests. Providers' refusal can go down to $-\infty$ because utilization can, theoretically, grow up to $+\infty$. In order to guarantee system stability, the way in which such intentions are computed is considered as private information for providers.

\section{$3 \quad \mathrm{~K}_{n}$ Best Method}

In this section, we present the $K_{n}$ Best method for balanced request allocation. It is meant to be run by one or several mediators which allocate the requests they receive from the clients. We restrict ourselves to the case where requests can be viewed as single units of work called tasks. An incoming feasible request $q$ can be allocated to $n$ providers (because of data heterogeneity, more than one provider can provide answers). We assume that there is a matchmaking mechanism ([8] for example) to find the set $P_{q}$ of providers that are able to deal with each incoming feasible request $q$. Therefore, we can focus on request allocation only. Our method is inspired by the two random choices paradigm $[10,20,21]$. The principle of two random choices is to randomly select a set of providers $K$ among the $N_{q}$ providers and then allocate the request to the least utilized provider in $K . K_{n}$ Best uses a similar principle. We describe below the principle and properties of the $K_{n}$ Best. 


\section{1 $\mathrm{K}_{n}$ Best Principle}

Given an incoming feasible request $q$ and the set $P_{q}$ of providers which are able to deal with it, we denote the providers' intention for dealing with $q$ by the vector $\overrightarrow{P I}^{q}$. Algorithm 1 shows the main steps of $K_{n}$ Best for allocating $q$.

First (line 2 of Algorithm 1), a set $K$ of providers are selected at random among the set $P_{q}$, where $\|K\|=k$ and $k \in \mathbb{N}^{*}$. Second (line 3), the $k_{n} \in \mathbb{N}^{*}$ least utilized providers (i.e. the $K_{n}$ set) are selected among the set $K$ of providers ${ }^{1}$. Third (line 4 ), the providers' intention vector, $\overrightarrow{P I}^{q}[p]$, for dealing with $q$ is computed. Considering our system architecture [15], this operation is realized at the mediator sites and do not impact the network traffic. Next (line 5), the intentions' ranking vector $\vec{R}^{q}$ is computed. This ranking is necessary for the selection of providers to deal with $q$. Intuitively, $\vec{R}^{q}[1]$ is the most interested provider to deal with $q, \vec{R}^{q}[2]$ the second, and so on until to $\vec{R}^{q}[N]$ which is the least interested. Finally (lines $6-8$ ), the request $q$ is allocated to the $n$ best providers. If $N<n$, the request is simply allocated to all $N$ providers without any further considerations. The second (line 3) and fourth (line 5) phases can be solved using a sorting algorithm. So, in the worst case, their complexity is $O\left(k \log _{2}(k)\right)$ and $O\left(k_{n} \log _{2}\left(k_{n}\right)\right)$, respectively.

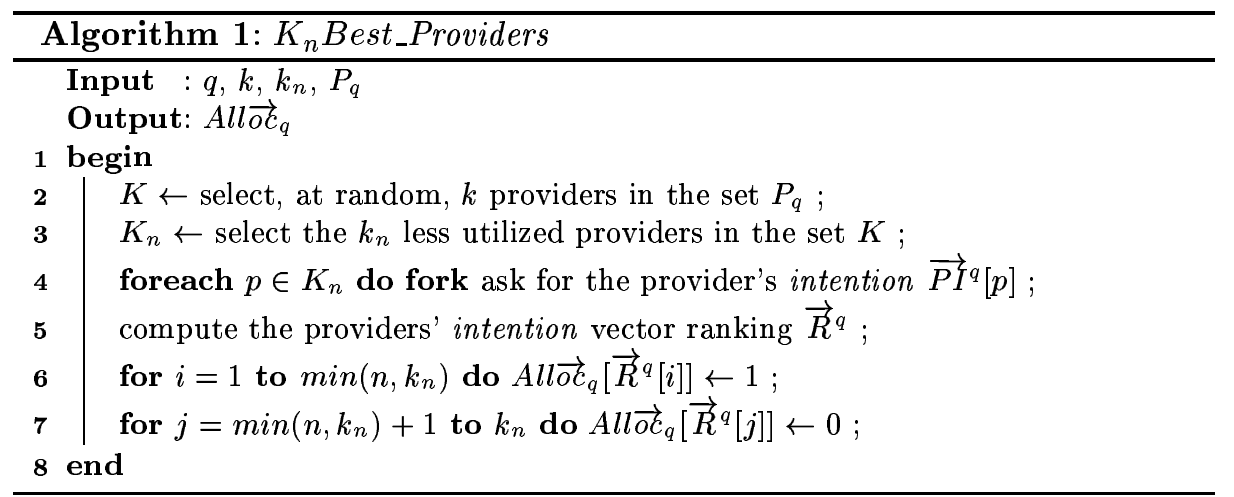

\section{2 $\mathrm{K}_{n}$ Best Property and Analysis}

An expected property of providers is individual rationality, whereby they behave according to their own interests only [1]. However, this may lead providers to not participate in a given request allocation since they are selfish and may have no interest in processing the request. Thus, individual rationality is incompatible with the system's objectives to process efficiently all incoming feasible requests and satisfy consumers. For these reasons, we are looking for a special kind of long-term individual rationality which $K_{n}$ Best allows.

\footnotetext{
${ }^{1}$ We can indifferently assume that the values of $k$ and $k_{n}$ are predefined by the
} administrator or defined on the fly by mediators. 
In the case of $m$ requests and $m$ homogeneous providers, request allocation methods based on the two random choices paradigm (which we call the TRCBased methods) assign to providers a maximum load of only $\theta(\log \log m)$ with high probability [10,20]. Given an incoming request $q, T R C B$ ased methods randomly select two providers (the $K$ set) among $P_{q}$ and allocate $q$ to $p \in K$, such that

$$
\forall p^{\prime} \in K \backslash \widehat{P_{q}}, U_{t}\left(p^{\prime}\right) \geq U_{t}(p)
$$

Nonetheless, the TRCBased methods are only suitable for homogeneous distributed systems (i.e. providers have the same capacity for performing requests). In the context of heterogeneous distributed systems, request allocation methods ensure good performances in the system by considering the capacities of all the providers in $P_{q}[6,12,14,17,21]$. These methods (the CapacityBased methods) allocate each incoming request $q$ to $p \in P_{q}$ such that

$$
\nexists p^{\prime} \in P_{q} \backslash \widehat{P_{q}}: U_{t}\left(p^{\prime}\right)<U_{t}(p)
$$

A third possible way to allocate requests is by only considering the providers' intentions, which we call the IntentionBased methods. Given the request $q$ to be allocated, IntentionBased methods allocate $q$ to $p \in P_{q}$ such that

$$
\nexists p^{\prime} \in P_{q} \backslash \widehat{P_{q}}: P I_{p^{\prime}}(q) \geq P I_{p}(q)
$$

On the other hand, the $k_{n}$ parameter defines the general behavior of the $K_{n}$ Best method. For smaller values of $k_{n}, K_{n}$ Best allocates the requests by mainly considering the providers' utilization, and by basically considering the providers' intentions for greater values of $k_{n}$. Thus, the value that $k_{n}$ may take depends on the type of application. Therefore, we recommend smaller values of $k_{n}$ if the system pays more attention to the providers' utilization and greater values if it pays more attention to the providers' intention. In the following theorem, we summarize the $K_{n}$ Best properties which bound its behavior.

Theorem 1. For a large number of heterogeneous providers, $K_{n}$ Best behavior is bounded by the following properties.

(i) if $k=2 q . n \wedge k_{n}=q . n, K_{n}$ Best is equivalent to the TRCBased methods.

(ii) if $k=N_{q} \wedge k_{n}=q . n, K_{n}$ Best is equivalent to the CapacityBased methods.

(iii) if $k=N_{q} \wedge k_{n}=k, K_{n}$ Best is equivalent to the IntentionBased methods.

Proof. Omitted because of triviality.

A different way to proceed in $K_{n}$ Best is by selecting first the most interested providers $\left(K_{n}\right)$ among $K$ to finally allocate requests to the least utilized providers among $K_{n}$. If request allocation is done this way, intentions are given more importance than utilization. Besides, one can replace the providers' intentions by the consumers' intentions for allocating requests if the objective of the system is, for example, to ensure interesting results for consumers. If the system's goal is to satisfy both providers and consumers, the consumers' intentions can be considered as an additional step in $K_{n}$ Best, or intentions of both providers and consumers can be merged in only one step. 


\section{Experimental Evaluation}

In this section, we give an experimental evaluation of $K_{n}$ Best using simulation. We carried out several series of tests with a main objective in mind: how well $K_{n}$ Best operates in environments with heterogeneous and autonomous providers. We assume a single mediator. In order to assess the quality of $K_{n} B e s t$, we conducted three types of evaluations: performance, request load balance, and satisfaction balance. In the following, we introduce the baseline methods to which we compare $K_{n} B e s t$ and our experimental setup, and then we present the experimental results.

\subsection{Baseline Methods}

In distributed information systems, the well-known methods to allocate requests efficiently across providers are those with the CapacityBased behaviour (i.e. the CapacityBased methods) $[6,14,17]$. These methods, unlike the TRCBased ones, operate well in heterogeneous systems which is why we use them as baseline.

Economic models have been shown to provide efficient request allocation in heterogeneous systems $[3,4,16,13]$. We use, as baseline, an Economic method whose criterion to select providers is the utilization and bid of providers. Note that different economic methods may have other performance results than those presented here. In our experiments, we use virtual money (token) which is just seen as a means of regulation. In the course of time, the tokens are spent by providers in order to acquire requests. Thus, a source of financing is necessary to them. Otherwise, after some time, providers would not have more tokens to bid positively. We have chosen to associate a bank with the mediator, which gives a specific amount of tokens to providers at the registration step and equally redistributes the tokens in the course of time.

\subsection{Experiment Setup}

We built a Java-based simulator that models a mono-mediator distributed system, following the system architecture in [15]. In all experiments, the number of consumers and providers is 200 and 400 respectively, with only one mediator allocating all the incoming feasible requests. We assign sufficient resources to the mediator so that it does not cause bottlenecks in the system. Each result we present is obtained by 10 simulation series. Feasible requests arrive to the system following the Poisson distribution, which has been found in dynamic and heterogeneous distributed systems [5].

For our simulations, we consider that providers provide answers with similar quality, hence assuming that consumers always ask for 1 provider to solve their requests (i.e. $q . n=1)$. Since we focus on heterogeneous distributed systems, we set $k=N_{q}$. Basing ourselves on the results of $[10,20]$, we set $k_{n}=2 q . n$ (i.e. $k_{n}=2$ ). In order to compare the $K_{n}$ providers, we consider their intentions for performing requests. Providers work out their intentions using the $S_{b} Q L B$ method [7]. To ensure high autonomy in our experiments, providers' preferences 
Table 1. Simulation parameters.

\begin{tabular}{lll}
\hline Parameter & Definition & Value \\
\hline nbConsumers & Number of consumers & 200 \\
nbProviders & Number of providers & 400 \\
nbMediators & Number of mediators & 1 \\
qDistribution & Query arrival distribution & Poisson \\
iniSatisfaction & Initial providers' satisfaction & 0 \\
qClasses & Supported query classes & 2 \\
nbSimulations & Number of realized simulations for each experience test & 10 \\
\hline
\end{tabular}

are randomly obtained between -1 and 1 . More sophisticated mechanisms for obtaining such preferences can be applied ([2] for example).

Since our main focus in this paper is to study the way in which requests are allocated in the system, we do not take into account the bandwidth problem. It is because of this that simulation tests are enough for evaluating our method. Providers are initialized with a satisfaction value of 0 . We set the providers' capacity heterogeneity in accordance to the results in [18]. Based on these results, we generate around $10 \%$ of low-capable, $60 \%$ of medium-capable, and $30 \%$ of highly-capable providers. The highly-capable providers are 3 times more capable than medium-capable providers and still 6 times more capable than low-capable ones. We generate two classes of requests that consume, respectively, 130 and 150 computational units at the high-capable providers (taking about 1.3 and 1.5 seconds, respectively).

\subsection{Experimental Results}

Performance. We start with an evaluation of the system's response time guaranteed by the CapacityBased, Economic, and $K_{n}$ Best methods. As is conventional, we define the response time as the elapsed time from the time a consumer issues the request to the time it receives the answer. As first case, we consider that providers are captive and do not leave the system by unsatisfaction, request starvation, nor overutilization. Figure 1 (a) shows that $K_{n}$ Best yields the same response times as CapacityBased, and that both of them outperform the Economic method. This shows that $K_{n}$ Best is also suitable for environments where providers do not leave the system.

As second case, in order to evaluate the impact of provider departures, we study response time when providers may leave the system by unsatisfaction or request starvation. To do so, we allow providers to leave the system if their satisfaction is 0.15 under their adequation. The adequation notion (see [7] for details) denotes the degree of satisfaction towards all the feasible requests proposed by the system. Also, providers are allowed to leave the system if they do not perform at least $25 \%$ of what they should. The results are shown in Figure 1(b). We observe that $K_{n}$ Best significantly outperforms the CapacityBased and Economic methods. While $K_{n}$ Best degrades in average the system's response time by a factor of 1.5 only, the Economic and CapacityBased do it, respectively, 


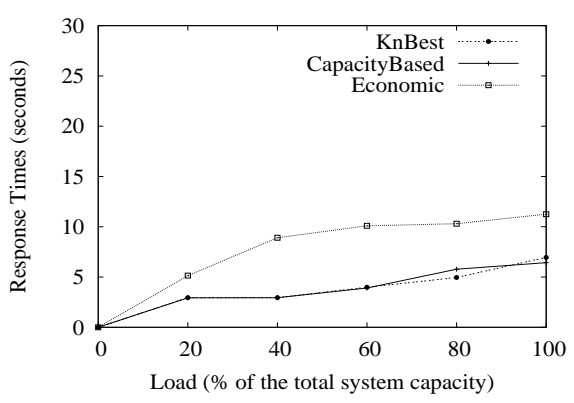

(a) Providers do not leave the system

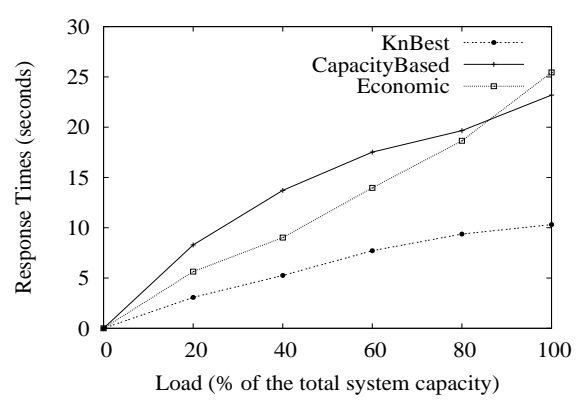

(b) Providers may leave the system

Fig. 1. Response Time.

by a factor of 1.8 and 4 ! We observed that in the Economic method, providers usually quit by request starvation, while in the CapacityBased, they do so by unsatisfaction.

We ran other experiments where providers may also leave the system by overutilization $^{2}$, but by lack of space, we do not present the results here. We observed that $K_{n}$ Best and CapacityBased do not suffer from providers' departures by overutilization, while the performance of the Economic method is degraded by a factor of 4.5 and, worse again, at a given time the system remains with no providers! These results demonstrate the great impact on response times of providers departures by unsatisfaction, request starvation, and overutilization. Therefore, in a system where providers are selfish, we must pay special attention to their interests.

Request Load Balance. We now study the request load balance $(R L B)$ for various workloads. In the experiments, we measure the $R L B$ at any time as the ratio of the smallest and largest utilized providers. Furthermore, it is important that the system strives to give requests to all providers in the system if possible so they don't leave. Thus, we also measure the average request load balance $(\operatorname{avg} R L B)$ at a discrete time interval $\left[t_{1}, t_{2}\right]$. The $\operatorname{avg} R L B$ measure is symmetrical to the $R L B$ measure. The average utilization, $U_{\left[t_{1}, t_{2}\right]}(p)$, is said to be the average utilization of the provider $p$ at the discrete time interval $\left[t_{1}, t_{2}\right]$.

We know that the thresholds of request starvation and overutilization over which providers decide to leave, are very subjective and might depend on several external factors. Thus, to avoid any question on the choice of such thresholds, we assume, in this study, that providers are not allowed to leave the system whatever their degree of request starvation and overutilization are.

\footnotetext{
${ }^{2}$ For instance, when providers want to guarantee a good quality of service.
} 


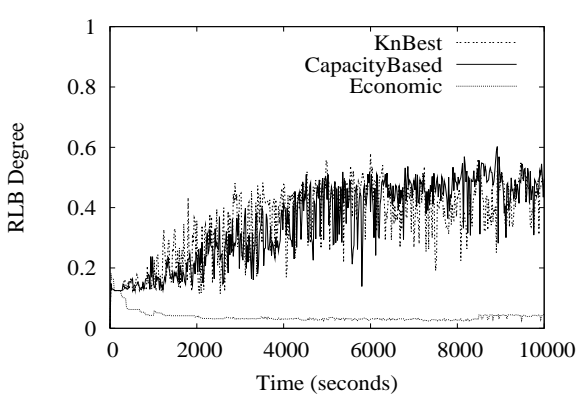

(a) For a workload from 30 to $100 \%$

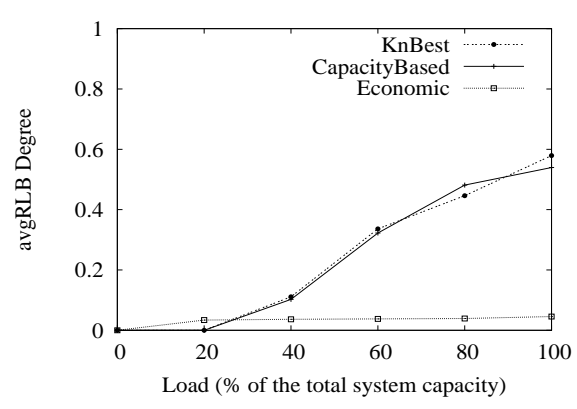

(b) In average for different workloads

Fig. 2. Request load balance.

Contrary to the expected, on the one hand, the results show that the Economic method has serious problems to ensure good $R L B$ ratios in the system, despite that providers take more into account their preferences than their utilization at the time of bidding for requests. On the other hand, the results show that CapacityBased and $K_{n}$ Best have problems to ensure good $R L B$ only for workloads under the $40 \%$ of the total system capacity. When workloads increase, both algorithms improve the $R L B$ in the system. This is because most providers in the first case have almost no requests (i.e. have all their capacity available), and thus, requests may be allocated to those providers that spend more treatment units to perform them. Hence, each time that requests are allocated to the least capable providers, the distance between the most and least utilized providers increases significantly. For lack of space, we do not present these results.

In order to explain the above phenomenon, we present the results of a series of simulations where we uniformly vary the workload from $30 \%$ (at the beginning of the simulation) to $100 \%$ (at the end of the simulation). The results show that CapacityBased and $K_{n}$ Best methods effectively improve the $R L B$ as the workload increases, and that the Economic method cannot ensure an acceptable $R L B$ in the system (see Figure 2(a)). In all cases the $K_{n}$ Best performance is as good as CapacityBased one, even if the former takes into account the providers' intentions and the latter does not.

Let us analyze the avgRLB guaranteed by these three methods, that is, how well these methods avoid request starvation. To this end, we measure the avgRLB in a time interval of 20 seconds over a simulation of 10000 seconds for different workloads. The results are shown in Figure 2(b). We observe that, unlike the $R L B$ results, for workloads over $20 \%$ of the total system capacity the CapacityBased and $K_{n}$ Best methods significantly outperform the Economic method. This means that while CapacityBased and $K_{n}$ Best strive to allocate requests on giving requests to all the providers in the system, the Economic method suffers from serious request starvation problems. 


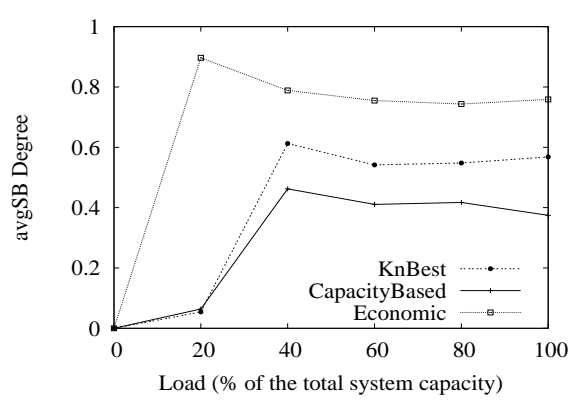

(a) Average satisfaction mean

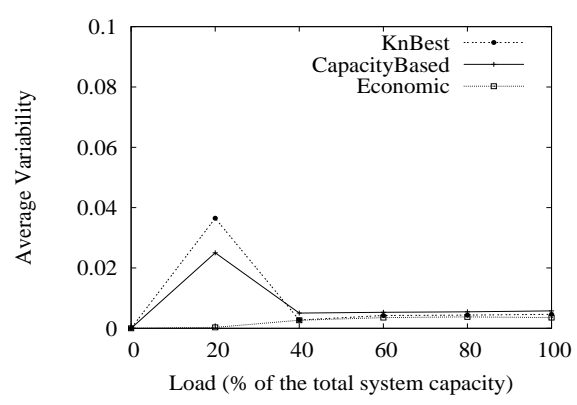

(b) Average satisfaction variability

Fig. 3. Providers' satisfaction in average.

Satisfaction Balance. We now study the satisfaction balance $(S B)$ ratios guaranteed by the three methods for various workloads. We assume that providers work out their satisfaction as in [7], but without loss of generality, providers can do it differently. For lack of space, we only present the average satisfaction balance $(\operatorname{avg} S B)$ measures, at a discrete time interval $\left[t_{1}, t_{2}\right]$. The avg $S B$ is defined as the ratio of the smallest and largest average satisfied providers, where average satisfaction denotes the average of the providers' satisfaction at the discrete time interval $\left[t_{1}, t_{2}\right]$. Furthermore, we measure the standard deviation of the providers' average satisfaction (average variability), in order to evaluate how these methods satisfy all providers. Similarly to the thresholds of request starvation and overutilization, the unsatisfaction threshold over which providers decide to leave, is also quite subjective and may depend on several external factors. Then, to avoid any question on the choice of such a threshold, we assume that providers are not allowed to leave the system by unsatisfaction.

Conversely to the $R L B$ results and as expected (because the providers' bids are based on the providers' intentions), the Economic method preserves better the providers' intentions (see Figure 3(a)). But this occurs because Economic does not pay much attention to the providers' utilization. Hence, this method is only suitable for systems where providers do not care about their utilization nor response time is very important for consumers. In these results, we have also observed that even if $K_{n}$ Best ensures the same $R L B$ than CapacityBased, it significantly satisfies better providers. Nevertheless, we can observe that both methods have some difficulties to preserve the providers' intentions for a very low workload ( $20 \%$ the total system capacity). This is due to the fact that the number of incoming feasible requests is not enough for giving requests to all providers, and thus in both methods, requests are allocated to the least utilized providers. Note that $K_{n}$ Best can be improved in two ways: 1) setting the $k_{n}$ to a greater value, or 2) in selecting first the $k_{n}$ most interested providers and allocating requests to the $q . n$ least utilized ones in $K_{n}$. 
Figure 3(b) shows that, for workloads under $20 \%$ of the total system capacity, some providers get more satisfied than others in the CapacityBased and $K_{n}$ Best methods. This is why the average variability of both methods is high. However, for higher workloads, the three methods yield almost the same average variability.

\section{Related Work}

In the context of large-scale distributed information systems, most of the work on request allocation $[6,14,17,20,21]$ has dealt with the problem of allocating requests to the most capable providers. However, preserving providers' autonomy for performing requests has not received much attention. Economical methods $[3,4,16,13]$ strive to deal with such autonomy. In such models, every provider tries to maximize its revenue by selling services to consumers. Mariposa [13] pioneered the use of an economical method for dealing with the request allocation problem in distributed systems. In Mariposa, all the incoming requests are processed by a broker site, which given an incoming request, requests providers for bids. Providers bid for acquiring the request based on a local bulletin board. Then, the broker site selects a set of bids that has an aggregate price and delay under a bid curve provided by the consumer. Nevertheless, it is unclear if this method ensures good response times in adequacy with the providers' intentions. Furthermore, some requests may not get processed although it exist providers able to deal with it. Recently we have proposed the $S_{b} Q L B$ approach [7] to balance requests across providers while considering their intentions for performing requests. $S_{b} Q L B$ strives to balance requests in the course of time thereby reducing request starvation in the system. However, $K_{n} B e s t$ is orthogonal and more general than all these methods. The $K_{n}$ Best method is actually a set of solutions for different environments and types of applications.

\section{Conclusion}

In this paper, we addressed the problem of request allocation in large-scale distributed information systems with autonomous providers. We proposed the $K_{n}$ Best method which allocates requests by considering providers' intentions for performing requests in addition to their utilization. The principle of $K_{n}$ Best is similar to the two random choices paradigm which has proven useful for dynamically assigning tasks to providers. We described the implementation of $K_{n} B e s t$ in different scenarios with the different strategies to adopt. We gave an experimental evaluation which compares $K_{n}$ Best to traditional request allocation methods. We demonstrated through experimentation that CapacityBased and Economic are not suitable for distributed information systems where providers may leave by request starvation, overutilization, or unsatisfaction. The experimental results show that, with autonomous providers in the system, our method significantly outperforms these methods. $K_{n}$ Best is suitable for dynamic, very 
large-scale distributed information systems with competitive or cooperative behaviors. Our method is general and simple, so that it can be easily incorporated in existing distributed information systems. It generalizes traditional methods and can be adapted to the application by varying several parameters.

\section{References}

1. O. Morgenstern and J. von Neumann: Theory of Games and Economic Behavior. Pinceton University Press, Inc. 1980.

2. A. Sah, J. Blow, and B. Dennis: An introduction to the Rush language. In Procs. of the TCL Workshop. 1994.

3. D. Ferguson, C. Nikolaou, J. Sairamesh, and Y. Yemini: Economic Models for Allocating Resources in Computer Systems. Market-based control: a paradigm for distributed resource allocation. World Scientific Publishing Co., Inc. 1996.

4. D. Ferguson, Y. Yemini, and C. Nikolaou: Microeconomic Algorithms for Load Balancing in Distributed Computer Systems. In Procs. of the ICDCS Conference. 1988.

5. E. P. Markatos: Tracing a Large-Scale Peer to Peer System: An Hour in the Life of Gnutella. In CCGRID Symposium. 2002.

6. H. Zhu, T. Yang, Q. Zheng, D. Watson, O. Ibarra, and T. Smith: Adaptive Load Sharing for Clustered Digital Library Servers. In HPDC Symposium. 1998.

7. J.-A. Quiané-Ruiz, P. Lamarre, and P. Valduriez: Satisfaction-Based Query Load Balancing. In Procs. of the CoopIS Conference. 2006.

8. K. Sycara, M. Klusch, S. Widoff, and J. Lu: Dynamic Service Matchmaking Among Agents in Open Information Environments. In SIGMOD Record 28(1). 1999.

9. L. Li and I. Horrocks: A Software Framework for Matchmaking Based on Semantic Web Technology. In Procs. of the WWW Conference. 2003.

10. M. Mitzenmacher: The Power of Two Choices in Randomized Load Balancing. PhD. Thesis, UC Berkeley, 1996

11. M. Nodine, W. Bohrer, and A. Ngu: Semantic Brokering over Dynamic Heterogeneous Data Sources in InfoSleuth. In Procs. of the ICDE Conference. 1999.

12. M. Roussopoulos and M. Baker: Practical Load Balancing for Content Requests in Peer-to-Peer Networks. Distributed Computing 18(6):421-434. 2006.

13. M. Stonebraker, P. Aoki, W. Litwin, A. Pfeffer, A. Sah, J. Sidell, C. Staelin, and A. Yu: Mariposa: A Wide-Area Distributed Database System. In VLDB J. 5(1). 1996.

14. N. Shivaratri, P. Krueger, and M. Singhal: Load Distributing for Locally Distributed Systems. In IEEE Computer Journal 25(12). 1992

15. P. Lamarre, S. Cazalens, S. Lemp, and P. Valduriez: A Flexible Mediation Process for Large Distributed Information Systems. In Procs. of the CoopIS Conference. 2004.

16. R. Buyya, H. Stockinger, J. Giddy, and D. Abramson: Economic Models for Management of Resources in Grid Computing. In CoRR Journal. 2001.

17. R. Mirchandaney, D. Towsley, and J. Stankovic: Adaptive Load Sharing in Heterogeneous Distributed Systems. In Parallel and Distributed Computing J. 9(4). 1990

18. S. Saroiu, P. Krishna Gummadi, and S. Gribble: A Measurement Study of Peerto-Peer File Sharing Systems. In Procs. of the MCN Conference. 2002.

19. T. Özsu and P. Valduriez: Principles of Distributed Database Systems, (2nd ed.). Prentice-Hall, Inc. 1999.

20. Y. Azar, A. Z. Broder, A. R. Karlin, and E. Upfal: Balanced Allocations. In SIAM Journal on Computing 29(1). 1999.

21. Z. Genova and K. Christensen: Challenges in URL Switching for Implementing Globally Distributed Web Sites. In Procs. of the ICPP Workshops. 2000. 